\title{
Microfluidic and bio-applications of optical microfibres
}

\author{
G. Brambilla \\ Optoelectronics Research Centre, \\ University of Southampton, \\ Southampton, SO17 1BJ, U.K.
}

\begin{abstract}
This paper reviews the application of optical microfibers to microfluidics and bio-sensing.

\section{INTRODUCTION}

Optical microfibers (OMs) are optical fibre tapers with a micrometric or sub-micrometric uniform waist region (fig. 1).
\end{abstract}

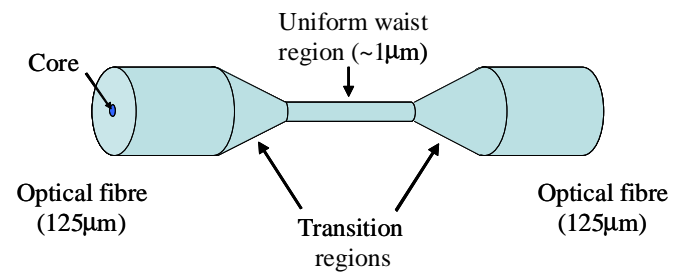

Fig. 1. Schematic of an optical microfiber (OM). The micrometric uniform waist region is connected to two optical fibre pigtails by conical transition regions.

OMs and related devices have attracted a continuously increasing attention [1], because of the numerous extraordinary optical and mechanical properties that they offer:

- biocompatibility; OMs are manufactured from silica fibres, thus they show all the physical properties of silica, including the good compatibility with cells and biological material in general.

- robustness: OMs exhibit a great mechanical strength, which allows for a relatively easy handling with micromanipulators and tools/equipment typical of the macroscopic world.

- flexibility: because of their small diameters, OMs have a small stiffness and can stand micrometric bending radii; increasing the device compactness.

- large evanescent fields: a considerable fraction of the transmitted power can propagate outside the OM physical boundary when the OM radius is small: the large evanescent field and its strong dependence on the adjacent environment can be exploited for sensing and optical manipulation.

- strong confinement: when an OM has a diameter comparable to half of the wavelength of the light transmitted in it, the propagating beam is confined by diffraction to its minimum waist diameter. If an OM tip is metal coated, the beam can be confined well beyond diffraction (to 50-100nm), allowing for sensing over extremely small areas.

- configurability: OMs are fabricated tapering optical fibres, thus they have a practically lossless connection (via conical transition regions) to their pigtails, which maintain the original optical fibre size and can be easily connected to other fiberized components.

Hereafter a summary of OM fabrication and properties will be presented, followed by an analysis of different typologies of applications to microfluidics and biology, including sensors, optical trapping and manipulation.

\section{FABRICATION AND PROPERTIES}

\section{A. Fabrication}

OMs have been manufactured mostly using one of the following four methods [1]:

1) Flame-brushing [2]

2) Modified flame-brushing [3]

3) Self-modulated taper-drawing [4]

4) Micropipette puller [5]

The vast majority of OMs reported in the literature have been manufactured using the "flame-brushing" technique, which was originally developed for the fabrication of optical fibre tapers and couplers and relies on a small flame which travels along an optical fibre under longitudinal stress. The profile of the tapered fibre can be controlled with an extreme accuracy by finely controlling the flame movement and the fibre elongation.

The modified flame-brushing technique replaces the flame with a different heat source: a microheater or a sapphire capillary tube heated by a $\mathrm{CO}_{2}$ laser beam. It has been used to manufacture OMs from compound glass optical fibres

The "self-modulated taper-drawing" is the technique which demonstrated the capability to manufacture extremely small tapers (down to $10 \mathrm{~nm}$ ) and it is a two-step process: firstly, a taper with a diameter of few micrometers is drawn from an optical fibre using the conventional flame brushing technique; then, the taper is broken into two pigtailed halves, and one of them is wrapped onto a hot sapphire rod and pulled to sub-micrometric diameters. The sapphire rod is heated by a flame positioned at a distance from the fibre, and it conveys the heat in regular manner into a small volume.

Micropipette puller is a commercially available instrument originally developed to manufacture micropipettes from glass capillaries: this process is bases on a $\mathrm{CO}_{2}$ laser source which heats a fibre stretched by two spring-loaded clamps. Although this technique cannot achieve a good taper shape control, it can provide tips with diameter as small as $50 \mathrm{~nm}$ and very steep profiles. 


\section{B. Beam confinement}

In OMs light is index guided [6] by the refractive index contrast between glass and the surrounding medium. The $\mathrm{OM}$ guiding properties are determined by the value of $\mathrm{V}$, defined as

$$
V=\frac{2 \pi}{\lambda} r N A
$$

where $\lambda$ is the wavelength, $r$ the OM radius, $\mathrm{NA}\left(=\sqrt{n_{O M}^{2}-n_{\text {surr }}^{2}}\right)$ the $\mathrm{OM}$ numerical aperture, $n_{O M}$ the $\mathrm{OM}$ refractive index and $n_{\text {surr }}$ the refractive index of surrounding environment.

Like for optical fibres, OMs experience single mode operation for $V<2.405$ and have maximum confinement for $V \sim 2$ [6]. As $V$ decreases below 2, the mode becomes less and less bound; because of diffraction, the beam spot size $\omega$ continuously expands until it becomes orders of magnitude larger than $r$ for $V<0.6$. Since for silica OMs at maximum confinement $\omega \sim \lambda / 3$ in air and $\omega \sim \lambda / 2$ in water, sub-100nm spot sizes cannot be achieved with visible/near-IR light. This limitation has been overcome by using coated tapers (generally with $\mathrm{Al}$ ), which exploit the evanescent field along the longitudinal direction. In metal coated tapers [7], when $r$ is smaller than $\sim \lambda / 6$, an evanescent field exists in the longitudinal direction of decreasing sections; this has been exploited to produce highly divergent light beams over extremely small apertures at the tip apex. Although this technique provided $\omega<100 \mathrm{~nm}$, the device transmission efficiency $T_{\varepsilon}$ was poor; indeed the field of the fundamental mode decays exponentially along the longitudinal direction: for a $20 \mathrm{~nm}$ aperture, $\mathrm{T}_{\varepsilon} \sim 10^{-8}$ [7].

\section{Evanescent field}

For $\mathrm{V}<<2$, in uncoated tapers a considerable fraction of the power propagates in the evanescent field outside the fiber. The fraction of power propagating in the evanescent field $\left(\eta_{E F}\right)$ depends on the ratio $\lambda / \mathrm{r}$ and on the refractive index of the surrounding medium $[6,8]$.

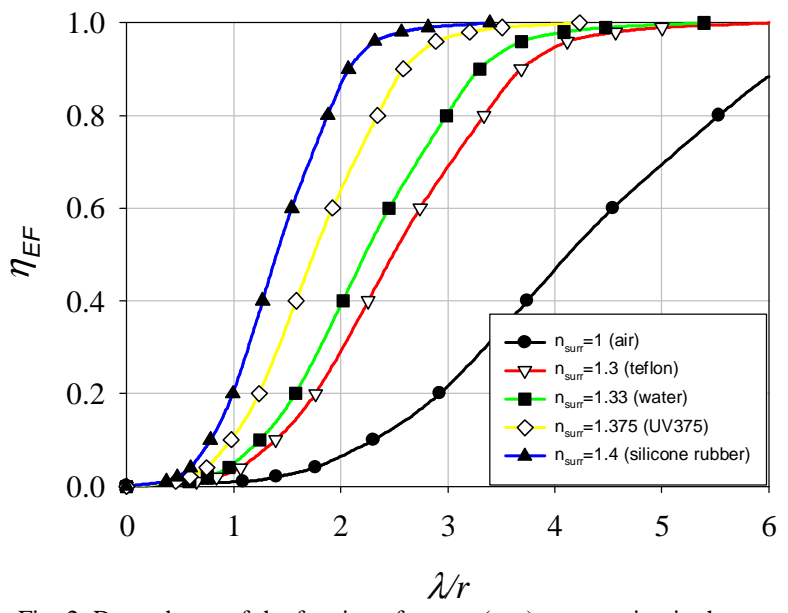

Fig. 2. Dependence of the fraction of power $\left(\eta_{E F}\right)$ propagating in the evanescent field of an OM on the normalised wavelength ( $\lambda$ is the light wavelength and $r$ the OM radius) for different surrounding media.
Fig. 2 shows the relation between $\eta_{E F}$ and $\lambda / \mathrm{r}$ for different types of surrounding materials, the refractive index of which $\left(\mathrm{n}_{\text {surr }}\right)$ varies between 1 and 1.4: $\eta_{E F}$ increases monotonically for increasing $\lambda / \mathrm{r}$ and it reaches $50 \%$ for $\lambda r \sim 4$ in air $(\mathrm{n} \sim 1)$ and for $\lambda / r \sim 2.4$ in water $(\mathrm{n} \sim 1.33)$. For $\lambda / r>5$ for most of dense surroundings, while for air, $\eta_{E F} \sim 1$ for $\lambda / r \sim 10$ [8].

\section{MICROFLUIDIC}

Because of the large fraction of power propagating in the evanescent field, OMs have been exploited for sensing in microfluidic channels. In fact, if the evanescent field overlaps with the microfluidic channel, a change in the composition and/or the refractive index of the fluid implies a change in the properties of the mode propagating in the OM.

By embedding an $\mathrm{OM}$ in a transparent low refractive index polymer (Sylgard 184, also called PDMS) in proximity of a fluidic channel [9], the evanescent field of the mode propagating in the OM overlaps with the microfluidic channel, thus the refractive-index difference between the polymer and the fluid strongly affects the OM transmission. The accuracy in the loss measurement affects the minimum measurable refractive index change, which resulted to be of the order of $\sim 5 \cdot 10^{-4}$. This is relatively small compared to refractometric biosensors based on plasmonics.

A more sensitive device has been proposed exploiting resonators. Resonators exhibit strong narrow dips in their transmission spectra. These dips are very sensitive to the surrounding conditions and shift their central wavelength when the surrounding refractive index is changed. By monitoring the dip wavelength shift, it is possible to determine the refractive index change in the surrounding environment. As for the tip sensors, functionalization can add selectivity to the sensors, inducing a refractive index change only for selected compounds.

Two different typologies of resonating devices have been realised: homogeneous and heterogeneous. In homogeneous resonating devices the resonator is fabricated by exploiting the OM modal self coupling given by the large evanescent field: by coiling an OM onto itself, modes propagating in two adjacent sections can overlap and couple, producing an extremely compact resonator. In heterogeneous devices OM is simply used to insert/extract light from the high-Q resonator manufactured by other means.

\section{A. Homogeneous resonators}

Three types of OM homogeneous resonators have been proposed: loop resonator [10], knot resonator [4] and microcoil resonators (MR) [11]. While loop and knot resonators are $2 \mathrm{D}$ systems, MR are truly $3 \mathrm{D}$ systems, where coupling between different turns occurs continuously along the whole length of the microcoil. Theoretically, MRs with larger numbers of turns offer higher Q-factors; still, when realistic losses are taken into consideration, three/four turn resonators exhibit $Q$-factor close to the practical limit. Experimentally, $Q$-factors as high as $10^{6}$ have been recorded. 


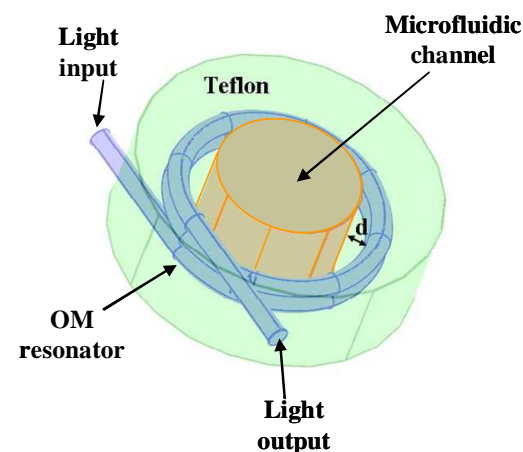

Fig. 3. Schematic of a microfluidic refractometric sensor based on OM microcoil resonators. The mode propagating in the $\mathrm{OM}$ has the evanescent field partly overlapping with the fluid flowing in the microfluidic channel.

Since MR can have an intrinsic channel (Fig. 3) which can be exploited for microfluidic applications, homogeneous refractometric sensors have been proposed [12] and demonstrated [13] using embedded MRs. Any change in the analyte refractive index is reflected in a shift in the resonant wavelength. The wavelength shift is strongly dependent on the ratio $r / \lambda$ and on the coating thickness $d$ between the MR and the fluidic channel. Sensitivities as high as $S=10^{3}$ $\mathrm{nm} / \mathrm{RIU}$ (refractive index unit) have been predicted for thin $d$. Experiments were carried out with an MR embedded in Teflon having a $1 \mathrm{~mm}$-wide microfluidic channel. The sensitivity of the device was tested by inserting the MR sensor in solutions of isopropanol and methanol and resulted to be $\sim 40 \mathrm{~nm} / \mathrm{RIU}$ because of the non-optimised design.

\section{B. Heterogeneous resonators}

Heterogeneous microfluidic resonators have been based on resonant modes in microcapillaries [14].

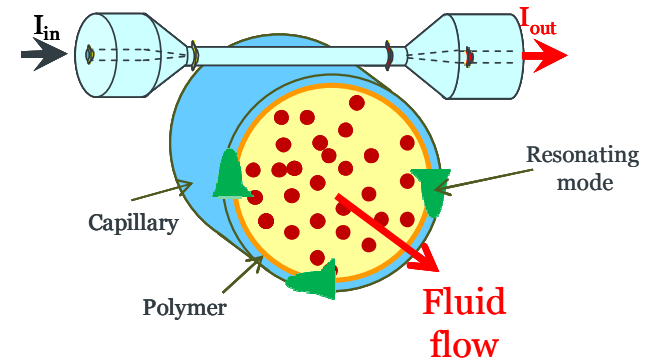

Fig. 4. Schematic of a heterogeneous sensor based on microcapillary. The mode propagating in the $\mathrm{OM}$ is coupled into the resonating mode of a microcapillary, the evanescent field of which overlaps with the fluid.

If an $\mathrm{OM}$ approaches a capillary orthogonally, it will excite a resonant mode which is bound to the capillary surface. If the capillary wall thickness is small, the mode will have a large overlap with the fluid flowing in it and it can be used for sensing. Liquid core optical ring-resonator (LCORR) sensors with $\mathrm{Q}>10^{5}$ and a sensitivity of several $\mathrm{nm} / \mathrm{RIU}$ have been demonstrated. By using capillaries with submicrometric wall thickness, S>390nm/RIU have been demonstrated. Using Ag nanoparticles and Rhodamine 6G dye as target molecule, SERS spectroscopy has been demonstrated in the microfluidic channel. The use of
Rhodamine 6G $1360 \mathrm{~cm}^{-1}$ Raman peak allowed to demonstrate detection below 410pM [15].

Functionalization with methyl phenol polysiloxane or polyethylene glycol allowed for the vapours of ethanol and hexane within 1s [16]. The flow rates used in this experiment were few considerably smaller than those of other optical vapour sensors: only $1 \mathrm{~mL} / \mathrm{min}$. Functionalization with M13specific antibodies allowed the detection of filamentous bacteriophage M13; vira concentration as small as $2.3 \cdot 10^{-3}$ $\mathrm{pfu} / \mathrm{ml}$ (pfu is the plaque-forming unit and it represents the number of infectious viruses) have been achieved.

\section{BIO-APPLICATIONS}

Bio-applications based on OM can be broadly classified in three groups: tip sensors, optical manipulation, heterogeneous resonant sensors.

\section{A. $\quad$ Tip sensors}

Bio sensors based on OM have exploited the extremely small end-face cross section (sub-200nm) to analyze minute areas, typically cell components. As explain in section II.B, in order to achieve extreme confinement, tip sensors were manufactured from metal coated OMs. The end face was left uncoated (fig. 5) and it underwent functionalization to facilitate covalent bonding of the targeted bio-molecule.

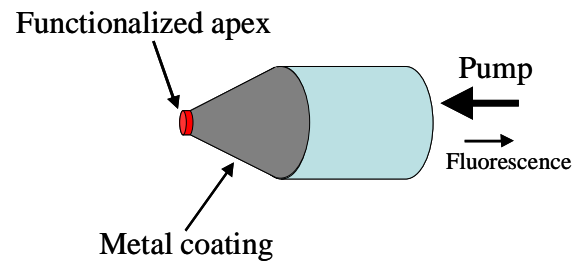

Fig. 5. Schematic of an OM tip sensor. Light injected at the fibre pigtail pumps the functionalised coating, which emits fluorescent light that is collected when it is in proximity of the target compound.

The functionalization compound is excited using the evanescent field generated inside the coated tip, thus only molecules in the immediate proximity of the OM apex aperture are affected by the optical field. Functionalization is generally carried out in three steps: silanization, activation and bonding of a biorecognition agent (usually antigens or antibodies with a fluorophore). The first OM employed for intracellular sensing was used to measure $\mathrm{pH}$ [17]. Functionalization was carried out using a chromophore (Nfluoresceinylacrylamide) in an acrylic copolymer. The chromophore was excited using blue light laser light and fluorescence was collected at 490, 540 and $610 \mathrm{~nm}$. pH in the range 4 to 9 was measured from the ratios between fluorescence peaks at different wavelengths $(540 / 490 \mathrm{~nm}$ and $540 / 610 \mathrm{~nm}$ ). The sensor response time was of the order of $\tau \sim 100 \mathrm{~ms}$. Biochemical compounds measured in-vivo, in live cells include benzopyrene tetrol and benzo[a]pyrene [18], cytochrome c [19], Caspase-9 [20], a telomerase [21] and DNA sequences [22]. The small sensor size allow for in-situ detection of specific chemicals without visible damage to the cell. DNA specific $\gamma$-actin mRNA strands were detected 
functionalising the apex with molecular beacons [22]; this method has been proved capable to recognise differences in single couple of bases. Ion concentrations were measured by functionalizing the sensor apex with plasticized polymeric membranes embedding ion complexation agents and fluorescent dyes. Potassium [20] and Calcium [23] concentrations were detected using valinomycin and Fura2/AM or fluo-3/AM calcium-dyes as highly-selective ligands. Other compounds measured in live cells include oxygen [24], nitrites [25], chloride ion [25] nitric oxide [26] and glutamate [27]. At the moment, the apex size is limited by the tip transmission efficiency. For tips smaller than $50 \mathrm{~nm}, \mathrm{~T}_{\varepsilon}<10^{-6}$ have been recorded [7]. A way to increase $\mathrm{T}_{\varepsilon}$ by orders of magnitude relies on the exploitation of surface plasmons: by converting light into surface plasmons and then back into light, confinements to spot sizes smaller than $10 \mathrm{~nm}$ should be possible [28], with extraordinary benefits for the sensor size.

\section{B. Optical manipulation}

Unlike tip sensors, which exploit the longitudinal evanescent component of a non-propagating field, optical manipulation exploits propagating fields. Massive cell manipulation [29] has been demonstrated on a planar waveguide exploiting the fraction of the mode propagating outside the waveguide physical boundary. This effect was explained as the contribution of two effects: 1) the gradient force, which attracts and traps the cells laterally and 2) the axial force due to photon scattering which propels cells along the direction of light propagation. Because of the large fraction of power propagating outside the OM physical boundary, OMs are particularly suited for this task. Indeed, OMs have been used to propel polystyrene microspheres with diameters in the range 3-10 $\mu \mathrm{m}$ [30] and microsphere clusters with diameters larger than $20 \mu \mathrm{m}$ [31]. A $500 \mathrm{~mW}$ fiberized Nd:YLF laser proved sufficient to propel microsphere along a sub- $\mu \mathrm{m}$ OM with speeds as high as $10 \mu \mathrm{m} / \mathrm{s}$ [31].

Single particle manipulation has been achieved cleaving an $\mathrm{OM}$ in the maximum confinement region. The propagating beam rapidly diverges and the intensity profile exhibits large gradients within very short distances. This provide the ideal conditions for the so-called optical tweezers. With respect to lensed optical fibres [32], which large sizes, difficult end face processing and large mode field diameters $(\sim 10 \mu \mathrm{m})$, OM decreases the level of power needed for trapping and allows for small probe dimensions and for sub-micrometric spot sizes [33]. $1 \mu \mathrm{m}$ silica microspheres in a water suspension were trapped at powers $\mathrm{P}>10 \mathrm{~mW}$, lower than $\mathrm{P} \sim 1 \mathrm{~W}$ used in free space [34] or $\mathrm{P} \sim 22 \mathrm{~mW}$ with lensed fibres [32].

\section{Heterogeneous resonant sensors}

Heterogeneous resonators represent a large group of OM bio-applications. This group of resonant sensors exploit OMs only to inject/extract light from high-Q resonators (like microspheres, microtoroids, microcapillaries and bottle resonators) which are in contact with a solution. Evanescent sensing in these types of high-Q resonators has been used to monitor chemical and biological elements positioned in proximity of the resonator surface. As for the case of homogeneous resonators (section III.A), the analyte alters the resonance wavelength of the resonator and by monitoring the wavelength shift it is possible to determine the analyte concentration with a high degree of accuracy. In general, high-Q factors are associated with very high sensitivities, but also with very difficult input/output coupling. OM have proved the best solution for coupling, providing efficiencies in excess of $90 \%$ [35].

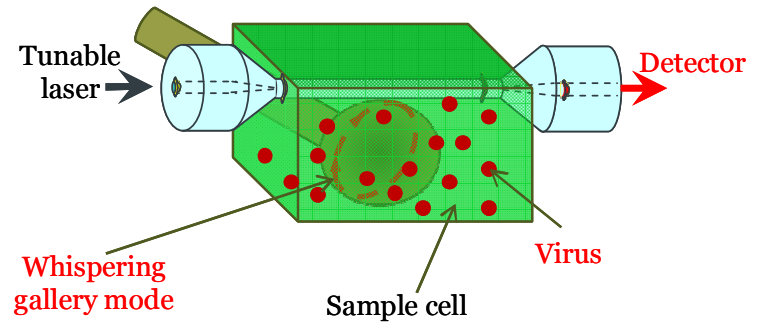

Fig. 6. Schematic of an heterogeneous resonant sensor. OM couples light into the resonant shispering gallery mode of a microsphere (dashed line), which is affected by the surrounding fluid.

Because of their high $\mathrm{Q}$ and of their extreme ease of fabrication, microspheres have been widely used for biological detection (fig. 6). Streptavidin was detected using a microsphere resonator with $\mathrm{Q} \sim 2 \cdot 10^{6}$ functionalised with bovine serum albumin biotin [36]. Specific DNA strands were detected using multiple spheres coated first with a dextran-biotin hydrogel and then with a mixture of biotinylated 27-mer oligonucleotides and streptavidin [37]. Single vira of Influenza $A$ and its mass ( 0.52 fg) were detected [38] by using a microsphere with radius of $39 \mu \mathrm{m}$ and light at a wavelength $\lambda=763 \mathrm{~nm}$. Single cylindrical bacteria were detected exploiting microsphere surface adsorption and the related resonance bandwidth broadening associated to increased scattering losses: detection limit of 44 bacteria was achieved for Escherichia Coli [39].

Toroidal microresonator are also popular because of their extremely high-Q. They have been used for detection of single molecules of interleukin-2 (a cytokine) [40] functionalizing the silica surface to bind the target molecule: by measuring the resonance wavelength shift, concentrations from $5 \mathrm{aM}$ to $1 \mu \mathrm{M}$ were recorded.

\section{ACKNOWLEDGMENT}

The author gratefully acknowledges the Royal Society (London, U.K.) for his Research Fellowship and EPSRC for partial financial support. He also thanks F. Xu, M. Ding, Y. Jung, P. Horak, G. S. Murugan, T. Melvin, J. Wilkinson, and D.J. Richardson for their contributions to research.

\section{REFERENCE}

[1] G. Brambilla, , "Optical fibre nanowires and microwires: a review," J. Opt., vol. 12(4), 043001, April 2010.

[2] G. Brambilla, V. Finazzi, D.J. Richardson, "Ultra-low-loss optical fiber nanotapers", Opt. Express, vol. 12, pp. 2258-63, 2004. 
[3] G. Brambilla, F. Koizumi, X. Feng, D.J. Richardson, Compoundglass optical nanowires", Electron. Lett., vol. 41, pp. 400-2, 2005.

[4] L.M. Tong, R.R. Gattass, J.B. Ashcom, S.L. He, J.Y. Lou, M.Y. Shen, I. Maxwell, E. Mazur, Subwavelength-diameter silica wires for low-loss optical wave guiding, Nature, vol. 426, pp. 816-9, December 2003

[5] K. T. Flaming and D. G. Brown, "Advanced Micropipette Techniques for Cell Physiology", Wiley Interscience, Chichester, 1986.

[6] L. Tong, J. Lou, E. Mazur, "Single-mode guiding properties of subwavelength-diameter silica and silicon wire waveguides", Opt. Express, vol. 12, 1025-35 (2004).

[7] B. Hecht, B. Sick, U.P. Wild, V. Deckert, R. Zenobi, O.J.F. Martin, D.W. Pohl, "Scanning near-field optical microscopy with aperture probes: Fundamentals and applications", J. Chem. Phys., vol. 112, pp. $7761-74,2000$

[8] G. Brambilla, Optical fibre nanotaper sensors, Opt. Fib. Technol., 2011, in press,

[9] P. Polynkin, A. Polynkin, N. Peyghambarian, M. Mansuripur, "Evanescent field-based optical fiber sensing device for measuring the refractive index of liquids in microfluidic channels", Opt. Lett., vol. 30, pp. 1273-5, 2005.

[10] M. Sumetsky, Y. Dulashko, A. Hale, "Fabrication and study of bent and coiled free silica nanowires: Self-coupling microloop optical interferometer", Opt. Express, vol. 12, pp. 3521-31, 2004.

[11] M. Sumetsky, "Optical fiber microcoil resonators", Opt. Express, vol. 12, pp. 2303-16, 2004

[12] F. Xu, P. Horak, G. Brambilla, "Optical microfiber coil resonator refractometric sensor", Opt. Express, vol. 15, pp. 7888-93, 2007.

[13] F. Xu, G. Brambilla, "Demonstration of a refractometric sensor based on optical microfiber coil resonator", Appl. Phys. Lett., vol. 92, 101126, 2008

[14] I. M. White, H. Oveys, X. Fan, "Liquid-core optical ring-resonator sensors", Opt. Lett., vol. 31, pp. 1319-21, 2006.

[15] I.M. White, J. Gohring, X. Fan, "SERS-based detection in an optofluidic ring resonator platform", Opt. Express, vol. 15, pp. 17433-42, 2007.

[16] Y. Sun, S.I. Shopova, G. Frye-Mason, X. Fan, "Rapid chemicalvapor sensing using optofluidic ring resonators", Opt. Lett., vol. 33, pp. 788-90, 2008.

[17] W. Tan, Z.Y. Shi, S. Smith, D. Birnbaum, R. Kopelman, "Submicrometer Intracellular Chemical Optical Fiber Sensors", Science, vol. 258, pp. 778-81, 1992

[18] T. Vo-Dinh, J.P. Alarie, B.M. Cullum, G.D. Griffin, "Antibodybased nanoprobe for measurement of a fluorescent analyte in a single cell", Nat. Biotechnol. Vol. 18, pp. 764-7, 2000.

[19] P.M. Kasili, J.M. Song, T. Vo-Dinh, Optical sensor for the detection of caspase-9 activity in a single cell, J. Am. Chem. Soc. 9, pp. 2799806, 2004.

[20] J.M. Song, P.M. Kasili, G.D. Griffin, T. Vo-Dinh, "Detection of Cytochrome c in a Single Cell Using an Optical Nanobiosensor", Anal. Chem., vol. 76, pp. 2591-4, 2004.

[21] X.T. Zhenga, C.M. Li, "Single living cell detection of telomerase over-expression for cancer detection by an optical fiber nanobiosensor", Biosens. Bioelectron., vol. 25, pp. 1548-52, 2010.

[22] X. Liu, W. Farmerie, S. Schuster, W. Tan, "Molecular Beacons for DNA Biosensors with Micrometer to Submicrometer Dimensions",
Anal. Biochem., vol. 283, pp. 56-63, 2000.

[23] J.D. Bui, T. Zelles, H.J. Lou, V.L. Gallion, M.I. Phillips, W. Tan, "Probing intracellular dynamics in living cells with near-field optics", J-Neurosci-Methods, vol. 89, pp. 9-15, 1999.

[24] Z. Rosenzweig, R. Kopelman, "Development of a Submicrometer Optical Fiber Oxygen Sensor", Anal. Chem., vol. 67, pp. 2650-4, 1995.

[25] S.L.R. Barker, B.A. Thorsrud, R. Kopelman, "Nitrite- and ChlorideSelective Fluorescent Nano-Optodes and in Vitro Application to Rat Conceptuses", Anal Chem, vol. 70, pp. 100-4, 1998.

[26] S.L.R. Barker, R. Kopelman, T.E. Meyer, M.A. Cusanovich, "FiberOptic Nitric Oxide-Selective Biosensors and Nanosensors", Anal. Chem., vol. 70, pp. 971-6, 1998.

[27] J. Cordek, X. Wang, W. Tan, "Direct immobilization of glutamate dehydrogenase on optical fiber probes for ultrasensitive glutamate detection", Anal. Chem., vol. 71, pp. 1529-33, 1999.

[28] F. Renna, D. Cox, G. Brambilla, "Efficient sub-wavelength light confinement using surface plasmon polaritons in tapered fibers", Opt. Express, vol. 17, pp. 7658-63, 2009.

[29] B. S. Ahluwalia, P. McCourt, T. Huser, O. G. Hellesø, "Optical trapping and propulsion of red blood cells on waveguide surfaces," Opt. Express, vol. 18, pp. 21053-21061, September 2010.

[30] G. Brambilla, G. S. Murugan, J. S. Wilkinson, D. J. Richardson, "Optical manipulation of microspheres along a subwavelength optical wire", Opt. Lett., vol. 32, pp. 3041-3043, 2007.

[31] G. S. Murugan, G. Brambilla, J. S. Wilkinson, D. J. Richardson "Optical propulsion of individual and clustered microspheres along sub-micron optical wires", Jap. J. Appl. Phys., Vol. 47(8), pp.67166718,2008

[32] K. Taguchi, K. Atsuta, T. Nakata, M. Ikeda, "Single laser beam fiber optic trap", Opt. Quantum Electron., vol. 33, pp. 99-106 2001.

[33] G. Brambilla, F. Xu, "Adiabatic submicrometric tapers for optical tweezers", Electron. Lett., vol. 43, pp. 204-206, 2007.

[34] A. Ashkin, "Optical trapping and manipulation of neutral particles using lasers", Proc. Natl. Acad. Sci. USA, Vol. 94, pp. 4853-4860, May 1997

[35] J.C. Knight, G. Cheung, F. Jacques, T.A. Birks, "Phase-matched excitation of whispering-gallery-mode resonances by a fiber taper", Opt. Lett., vol. 22, pp. 1129-31, 1997

[36] F. Vollmer, D. Braun, A. Libchaber, M. Khoshsima, I. Teraoka, and S. Arnold, "Protein detection by optical shift of a resonan microcavity", Appl. Phys. Lett., vol. 80, pp. 4057-9, 2002.

[37] F. Vollmer, S. Arnold, D. Braun, I. Teraoka, A. Libchaber "Multiplexed DNA Quantification by Spectroscopic Shift of Two Microsphere Cavities", Biophysical J., vol. 85, pp. 1974-9, 2003.

[38] F. Vollmer, S. Arnold, D. Keng, "Single virus detection from the reactive shift of a whispering-gallery mode", PNAS, vol. 105, pp. 20701-4, 2008

[39] H.C. Ren, F. Vollmer, S. Arnold, A. Libchaber, "High-Q microsphere biosensor - analysis for adsorption of rodlike bacteria", Opt. Express, vol. 15, pp. 17410-3, 2007.

[40] A.M. Armani, R.P. Kulkarni, S.E. Fraser, R.C. Flagan, K.J. Vahala, "Label-free, single-molecule detection with optical microcavities", Science, vol. 317, 783-6, 2007. 